\title{
Research Article \\ Simulation of Elliptical Liquid Jet Primary Breakup In Supersonic Crossflow
}

\author{
Yao-zhi Zhou $\mathbb{D}^{1,2}$ Feng Xiao $\mathbb{D}^{1,}{ }^{1,2}$ Qing-lian Li $\mathbb{D}$, ${ }^{1,2}$ and Chen-yang Li $\mathbb{D}^{1,2}$ \\ ${ }^{1}$ Science and Technology on Scramjet Laboratory, National University of Defense Technology, Changsha 410073, China \\ ${ }^{2}$ College of Aerospace Science and Technology, National University of Defense Technology, Changsha 410073, China
}

Correspondence should be addressed to Qing-lian Li; peakdreamer@163.com

Received 28 December 2019; Accepted 21 February 2020; Published 5 June 2020

Guest Editor: Wei Lin

Copyright ( 2020 Yao-zhi Zhou et al. This is an open access article distributed under the Creative Commons Attribution License, which permits unrestricted use, distribution, and reproduction in any medium, provided the original work is properly cited.

\begin{abstract}
The study of elliptical liquid jets in supersonic flow in a Mach 2.0 is performed numerically. The primary breakup process of the elliptical liquid jet is simulated for a Weber number 223, liquid/gas flux momentum 4.0. The aspect ratios of elliptical geometries are set to be $0.25,0.5,1,2$, and 5 . The results show a remarkable difference in liquid jet disintegration morphology at different aspect ratios. Under supersonic crossflow conditions, the elliptical liquid jet shows more breakup characteristics than the round liquid jet. As the aspect ratio grows, the penetration depth decreases. The elliptical liquid jet with $\mathrm{AR}=0.25$ has the largest penetration depth in all cases. Moreover, the round jet has a maximum spreading angle of $50.2^{\circ}$. The changing trends of the column breakup length both $x$ direction and $y$ direction are similar. The elliptical jet at a lower aspect ratio has a shorter breakup length due to the narrower windward area. The liquid jet has a pair of larger horseshoe vortex structure and a wider wake region at a higher aspect ratio. Two pairs of reversal vortex pairs with obvious characteristics can be observed in all the simulations.
\end{abstract}

\section{Introduction}

Liquid fuel is directly injected into a supersonic flow in a scramjet engine normally; it must atomize fully and mix well in milliseconds [1-3]. A lot of experiments about liquid jet breakup in high-speed gas have been studied in recent years [4-7]. It is a significant phenomenon that the atomization of liquid jet in a gaseous crossflow had a wide application in fields such as combustion in liquid rocket engine, gas turbine engines, or scramjets [8-13].

Obviously, there are three important physical processes that control atomization characteristics, the deformation of liquid column, the formation of surface waves, and the primary breakup [14-17]. To study these physical processes, one of the most convenient ways is to change the geometry of liquid orifices. The breakup mechanisms of liquid jets in still air of different orifice geometries have been studied in the last three decades [18-22]. An interesting conclusion been found that the liquid jets injected from a noncircular orifice had a shorter breakup time than a round orifice in a same orifice area. Among the many noncircular orifices, the elliptical orifices have attracted plenty of attentions. Compared with the round liquid jets, the surface energy of elliptical liquid jets is smallest normally in a same orifice area. After the study of trajectories of elliptical jets in a gas flow, a theoretical model was developed in the works of [23]. In this model, as the aspect ratio increases, the penetration depth decreases. The spatial distribution of elliptical jets was studied using an empirical correlation experimentally in [24]. The deformation of the liquid column has some significant differences at different aspect ratios. A hybrid model was improved to apply a liquid jet in gas flow in [25]. The cross-section of the liquid column underwent a process from a circle to an ellipse, and the liquid mass is converted to discrete droplets at the same time.

Though many researchers referred to the improvement of spray efficiency through a process from a circle to an ellipse in elliptical jets, the elliptical liquid jet in a supersonic crossflow has not been studied so far. The speed of the crossflow is usually supersonic in a scramjet. There are many complex surface waves ahead of the liquid column; the flow field near the liquid column is very chaotic. The significance of 


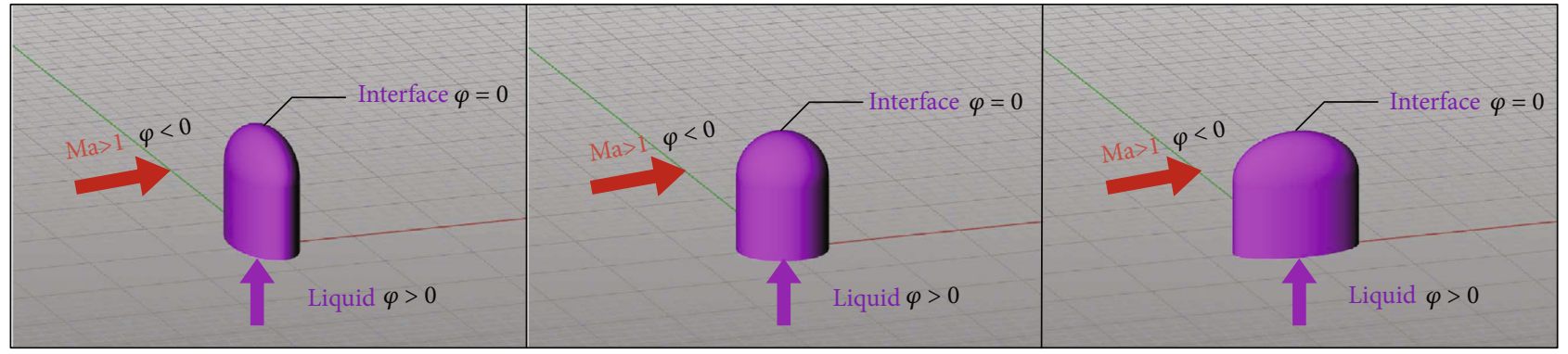

Figure 1: Initialization schematic between the round and elliptical jet.

TABLE 1: Supersonic crossflow conditions and liquid jet condition.

\begin{tabular}{|c|c|c|c|}
\hline \multicolumn{4}{|l|}{ Supersonic crossflow } \\
\hline Mach number & 2.0 & Gas velocity, $U_{\mathrm{G}}(\mathrm{m} / \mathrm{s})$ & 517.2 \\
\hline Static pressure, $P_{\mathrm{G}}(\mathrm{kPa})$ & 17.2 & Gas density, $\rho_{\mathrm{G}}\left(\mathrm{kg} / \mathrm{m}^{3}\right)$ & 0.3 \\
\hline Static temperature, $T_{\mathrm{G}}(\mathrm{K})$ & 167 & Viscosity coefficient, $\mu_{\mathrm{G}}(\mathrm{Pa} \cdot \mathrm{S})$ & $1.1 \times 10^{-5}$ \\
\hline \multicolumn{4}{|l|}{ Liquid jet } \\
\hline Injection velocity, $U_{\mathrm{L}}(\mathrm{m} / \mathrm{s})$ & 17.9 & Water density, $\rho_{\mathrm{L}}\left(\mathrm{kg} / \mathrm{m}^{3}\right)$ & 1000 \\
\hline Water pressure, $P_{\mathrm{L}}(\mathrm{kPa})$ & 160.2 & Momentum ratio, $q=\rho_{\mathrm{L}} U_{\mathrm{L}}^{2} / \rho_{\mathrm{G}} U_{\mathrm{G}}^{2}$ & 4.0 \\
\hline Surface tension coefficient, $\sigma(\mathrm{N} / \mathrm{m})$ & 0.072 & Viscosity coefficient, $\mu_{\mathrm{L}}(\mathrm{Pa} \cdot \mathrm{S})$ & $1 \times 10^{-3}$ \\
\hline
\end{tabular}

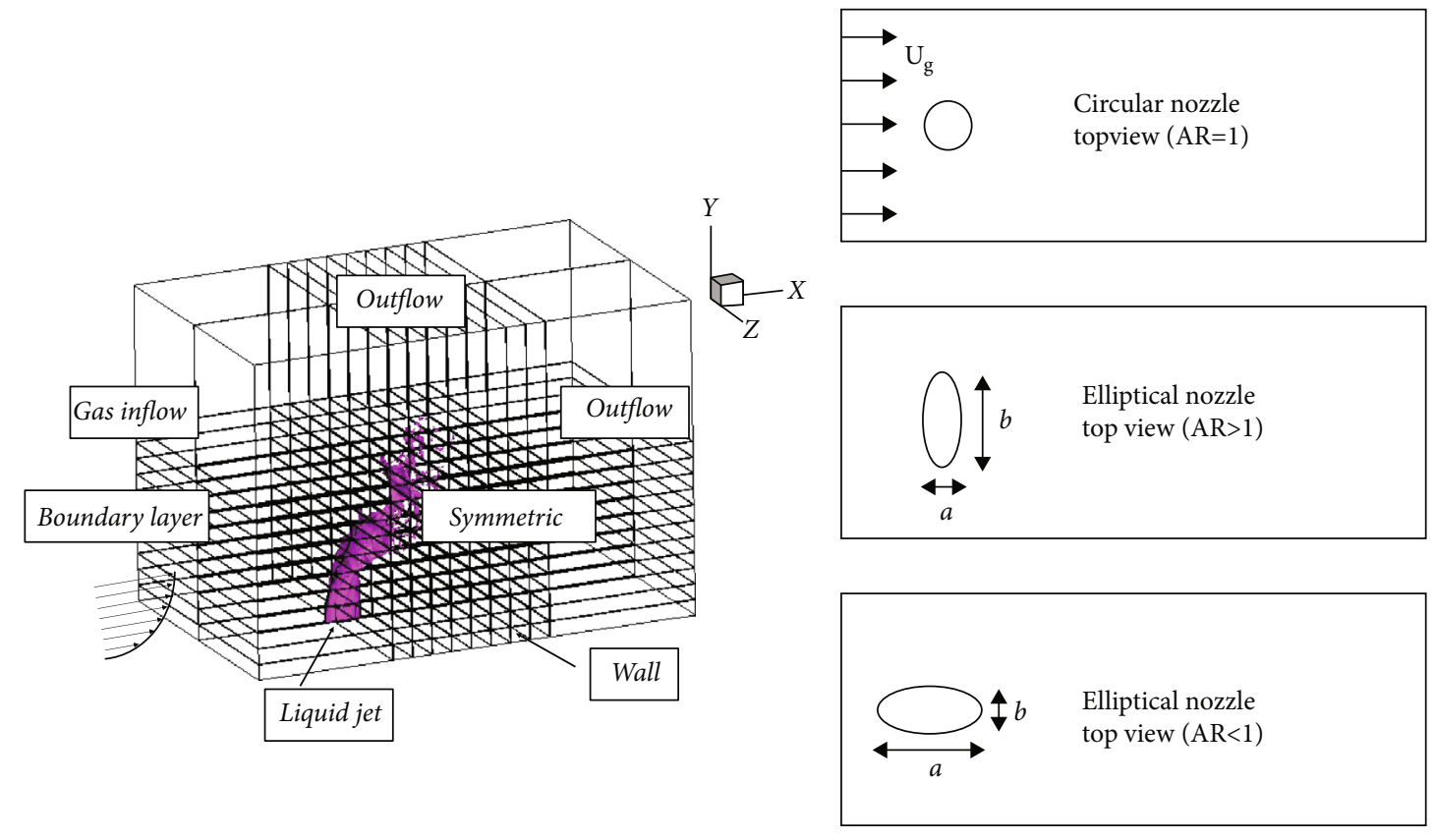

(a)

(b)

Figure 2: Simulation domain of elliptical liquid jet and top view of different orifice types.

this investigation focuses on the breakup processes of the elliptical jet, spatial distribution, and flow field characteristics presented in a supersonic crossflow which has not been fully investigated by previous studies.

Numerical research can obtain more flow field details than experimental research are applied in [26]. The detailed breakup process of a droplet in a supersonic flow has been studied numerically in [27-29]. The secondary atomization of a liquid jet has been predicted numerically in Ref. [30, 31]. Under the Euler-Lagrange framework, a two-phase simulation scheme was proposed in [32]. Under the EulerianLagrangian framework, the liquid trailing phenomenon of a liquid jet in a supersonic crossflow was captured using large eddy simulation (LES) in $[33,34]$. Using the CLSVOF method, the breakup process of a pulsed liquid jet in highspeed gas flow was studied in the works of [35]. 
TABLE 2: Geometries of injection nozzles (crossflow is from left to right).

\begin{tabular}{|c|c|c|c|c|c|c|c|}
\hline \multicolumn{2}{|c|}{ Configuration } & \multirow{2}{*}{$\begin{array}{c}\begin{array}{c}\text { Orifice } \\
\text { Geometry }\end{array} \\
\underset{\mathrm{a}}{\stackrel{--\mathrm{A}^{\prime}}{\longrightarrow}} \mathrm{b}\end{array}$} & \multirow{2}{*}{$\frac{a(\mathrm{~mm})}{0.4}$} & \multirow{2}{*}{$\frac{b(\mathrm{~mm})}{0.1}$} & \multirow{2}{*}{$\begin{array}{c}\mathrm{AR} \\
0.25\end{array}$} & \multirow{2}{*}{$\begin{array}{c}A\left(\mathrm{~mm}^{2}\right) \\
0.0314\end{array}$} & \multirow{2}{*}{$\frac{W e_{G}}{223}$} \\
\hline Case 1 & Elliptical & & & & & & \\
\hline Case 2 & Elliptical & $\underset{\mathrm{a}}{\longleftrightarrow}$ & 0.2828 & 0.1414 & 0.5 & 0.0314 & 223 \\
\hline Case 3 & Circular & $\underset{a}{4}$ & 0.2 & 0.2 & 1 & 0.0314 & 223 \\
\hline Case 4 & Elliptical & $\underset{a}{\underbrace{}_{a}}$ & 0.1414 & 0.2828 & 2 & 0.0314 & 223 \\
\hline Case 5 & Elliptical & $\mathrm{Mt}_{\mathrm{a}}$ & 0.1 & 0.4 & 4 & 0.0314 & 223 \\
\hline
\end{tabular}

TABle 3: Minimum mesh size under three kinds of meshes.

\begin{tabular}{lcc}
\hline Grid-A & Grid-C & Grid-F \\
\hline $0.045 D$ & $0.09 D$ & $0.03 D$ \\
\hline
\end{tabular}

This paper is organized as follows. The governing equations and numerical methods are described in Section 2. The breakup processes of the elliptical liquid jet in a supersonic crossflow are presented in Section 3.1; the spatial distribution of the elliptical liquid jet including the penetration depth, spreading angle, and breakup length is presented in Section 3.2. Then, Section 3.3 analyzes the flow field characteristics of the elliptical jet and round jet. Finally, a conclusion is presented in Section 4.

\section{Governing Equations and Numerical Method}

We adopted the CLSVOF method to track the liquid/gas interface. It absorbs the advantages of the LS method and the VOF method, which can capture the interface exactly and keep quality conservation for the two-phase flow in $[36,37]$. The VOF is defined as the volume fraction of liquid in every cell. The LS $\phi$ is a distance function; $\phi$ represents the signed distance from the interface. The isosurface $\phi=0$ represents the interface, with $\phi>0$ in liquid and $\phi<0$ in gas. Figure 1 depicts that the round jet has an initial liquid column during the initialization, while an elliptical jet has an initial elliptical cylinder, respectively. The detailed information about interface advection equations, liquid governing equations, and gas governing equations has been presented in Ref. [38, 39]. The spatially filtered LES formulation is employed in our solver, and the subgrid-scale (SGS) stress tensor arising from SGS eddies is modelled by the Smagorinsky eddy viscosity model. The temporal discretization and pressure gradient discretization can be referred to Ref. [38,

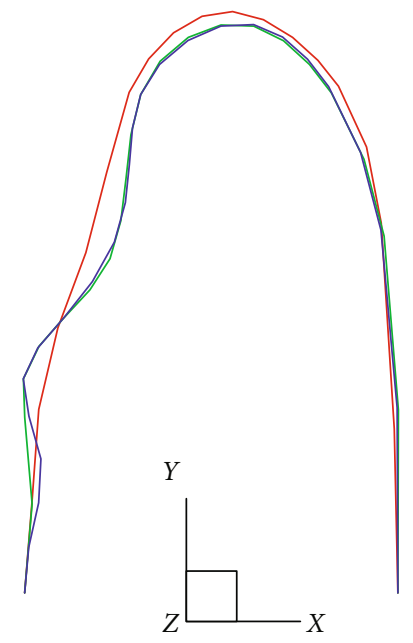

FIgure 3: Simulated initial column shapes on Grid-C (red line), Grid-A (green line), and Grid-F (blue line) for Case 1.

40-42]. Our solver has been validated with experimental results of a sphere in our previous works [38].

To study the effects of the elliptical orifice, five geometries of elliptical orifices have been run. And the gas conditions and liquid condition are given in Table 1. The simulation domain is divided into 240 blocks as shown in Figure 2(a), which is $[0,26 D] \times[0,15 D] \times[-8 D, 8 D]$ in the $x, y$, and $z$ directions. The equivalent diameter of the orifice outlet is represented by $D$, which is $0.2 \mathrm{~mm}$ in all the cases. A uniform Cartesian mesh is used in the region $[7.5 D, 16 D] \times[0,10 D]$ $\times[3.5 D, 3.5 D]$. The top and right faces are defined as outflow boundary conditions, and the front and back faces are defined as symmetric boundary conditions. Figure 2(b) shows the geometry shapes and the top view of different orifices. The geometries of injection orifices are given in Table 2 . 


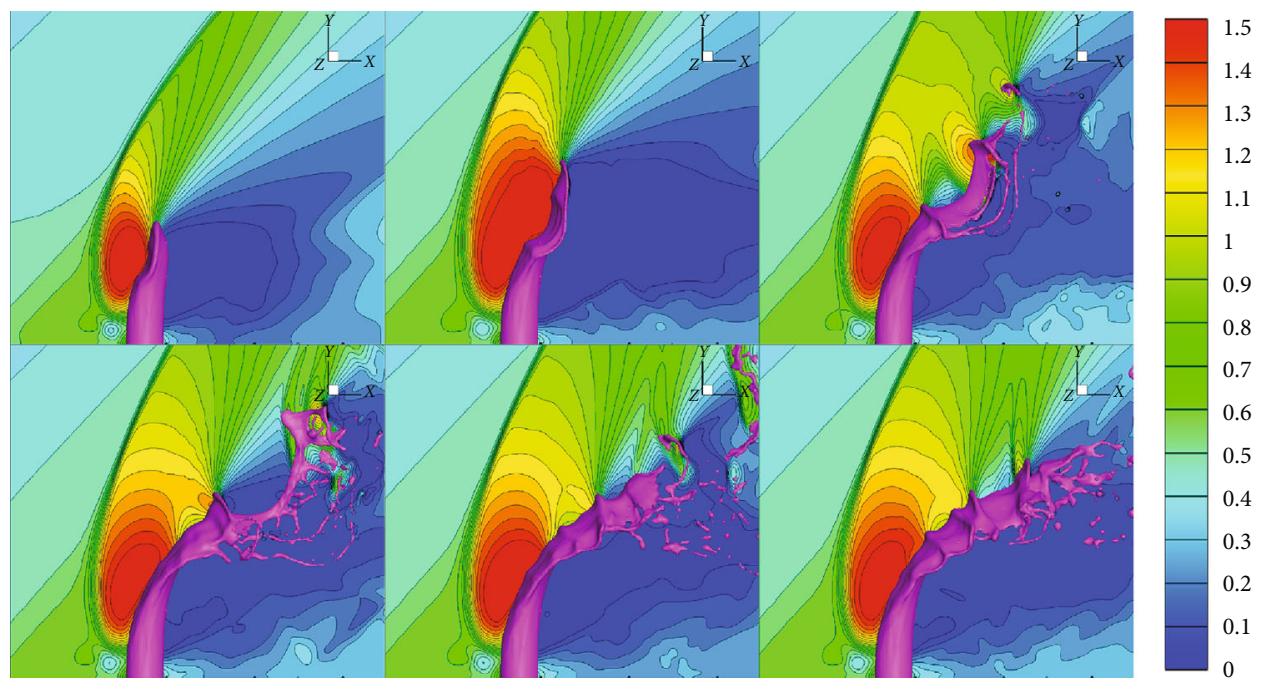

(a) Case 1

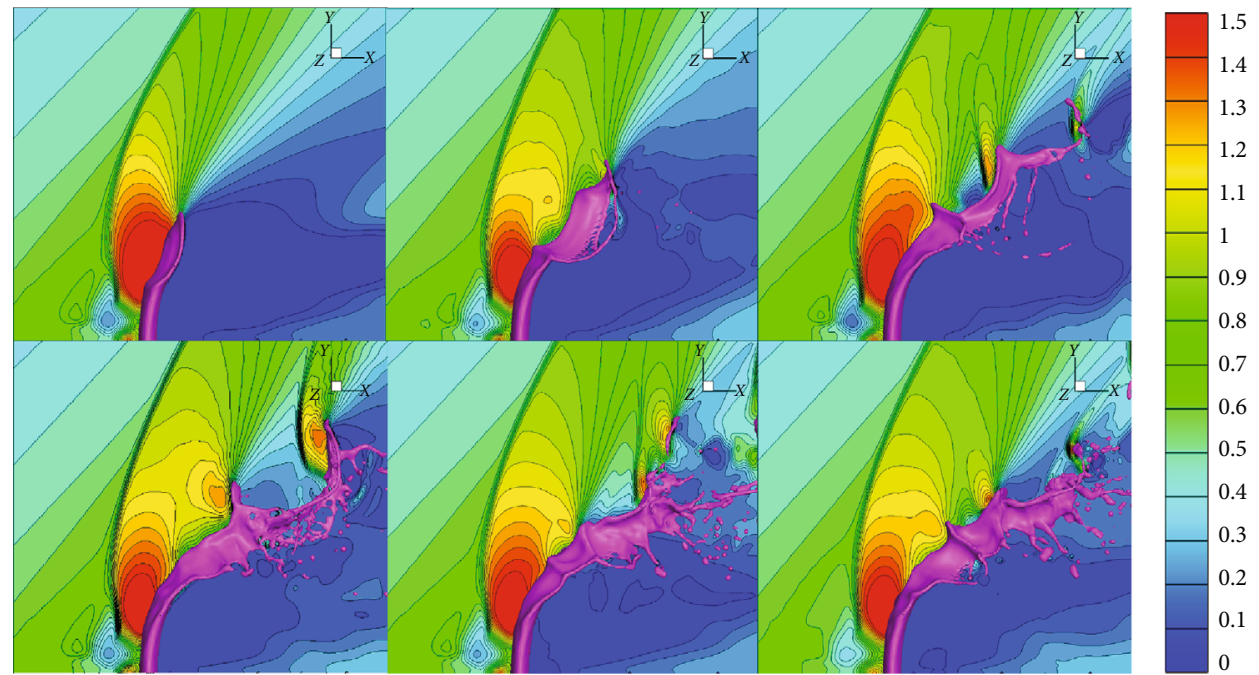

(b) Case 3

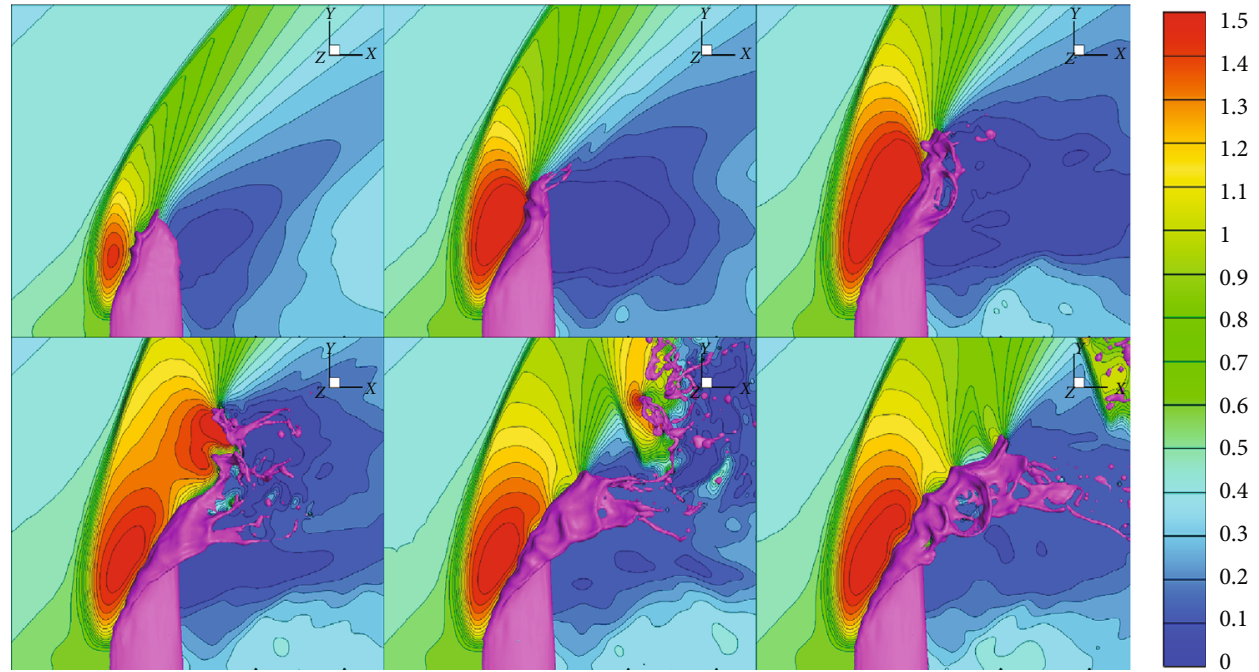

(c) Case 5

FIGURE 4: Morphology of liquid jet primary breakup with $P^{*}$ contours in slice $z=0$ for three AR, $\Delta t=8 \mu$ s. 


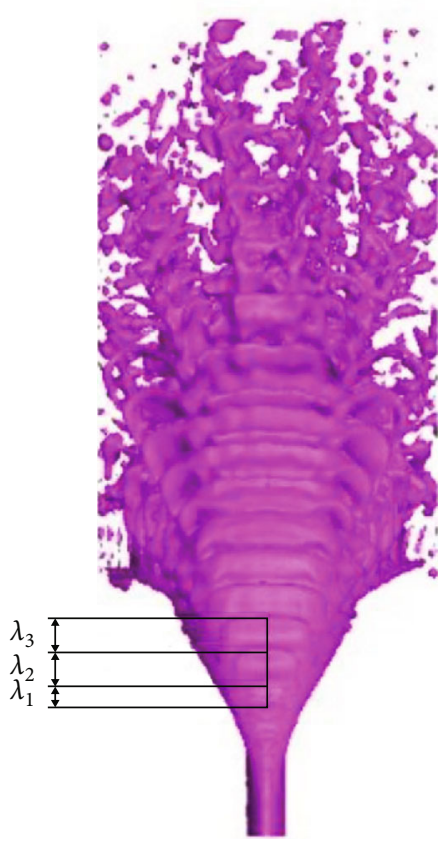

(a)

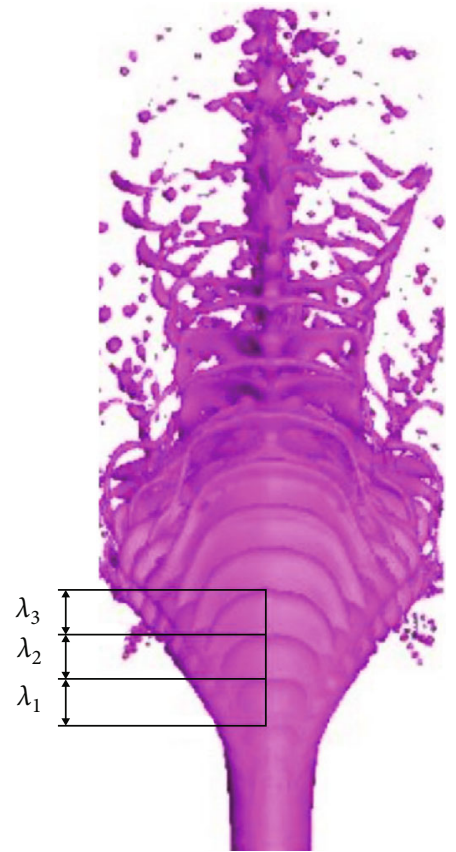

(b)

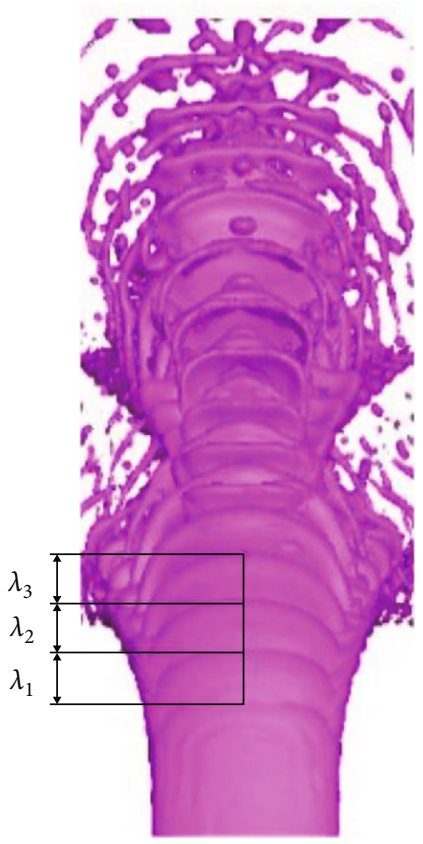

(c)

Figure 5: Measurement of surface wavelength for Case 1, Case 3, and Case 5.

Accordingly, a similar method has been used to check the grid resolution in Ref [38]. We used three different resolutions of the grid to simulate the cases in Table 3. There is a subtle difference between Grid-A and Grid-F in Figure 3. However, Grid-C predicts a significant interface shape compared with Grid-A and Grid-F. At last, the current grid (Grid-A) is chosen to be applied for all the cases in our works.

\section{Results}

3.1. Breakup Processes of Elliptical Liquid Jet in Supersonic Crossflow. Figure 4 shows the evolution of liquid jet breakup and the contours of the nondimensionalized pressure $\left(P^{*}\right)$ in the gas crossflow at the AR of $0.25,1$, and 4 . The difference of liquid column breakup process under these three aspect ratios is obvious, which will be taken as the main comparison condition in this paper. There is a bow shock wave forming ahead of the column. The primary breakup of the liquid jet includes two basic breakup modes: column breakup and surface breakup [2]. There are lots of ligaments stripping from the column in surface breakup mode. The liquid jet fractures on the central axis directly in column breakup mode. The length of $a$ becomes shorter with the increase of the AR, while the length of $b$ becomes longer with the increase of the AR at the same time, because of the same orifice area in all cases. Figure 5 shows that the wavelength of the initial surface waves becomes shorter with the decrease of the aspect ratio.

Figure 6 shows the cross-section of the jet at different locations, i.e., $y / D=1,2,2.5,3$, and 3.5. It can be seen that the liquid on the windward side pushes against both sides of the column in all calculated conditions. The column surface is peeled off from the liquid column due to the strong shear force. In the highest AR case, the droplet is fastest to peel off, and the jet is difficult to maintain the continuous liquid column shape and break up quickly, resulting in a chaotic section shape. However, in the working condition with the lowest AR, the jet can still retain a highly continuous column shape and have a relatively symmetric surface fracture due to the lower windward area. The airflow velocity on the leeward side is low, and the aerodynamic is not obvious. The morphology of the windside jet does not change too much; the jet can still maintain the shape of the initial column with a certain length after the surface breakage occurs. Based on the deformation law of a cross-section in present works in Figure 7, the windward area of the liquid jet is generally small when $\mathrm{AR}<1$, the pressure is larger on the stagnation point on the windward side, R-T instability dominates, and the liquid on the windward side is rapidly pushed to both left and right sides of the column and forms a pairs of reverse vortex pairs; When $A R=1$, the flow field under the twodimensional top view is similar to the flow field around a cylinder, which can be simply equivalent to the twodimensional flow around a cylinder for analysis. The KelvinHelmholtz instability and R-T instability exist simultaneously. The liquid column will still undergo pushing deformation. Compared with the cases $(\mathrm{AR}<1)$, the deformation occurs later, and the reverse vortex pairs are closer to the downstream region. When $\mathrm{AR}>1$, the windward area is generally larger. The aerodynamic force is larger at the left and right ends in the windward side of the jet. The surface breakup occurs earliest, and the influence of the $\mathrm{K}-\mathrm{H}$ instability is greater than the R-T instability at this time. However, when the surface breakup occurs, the windward area decreases rapidly, and the R-T instability gradually becomes dominant and forms pairs of reverse vortex pairs. 


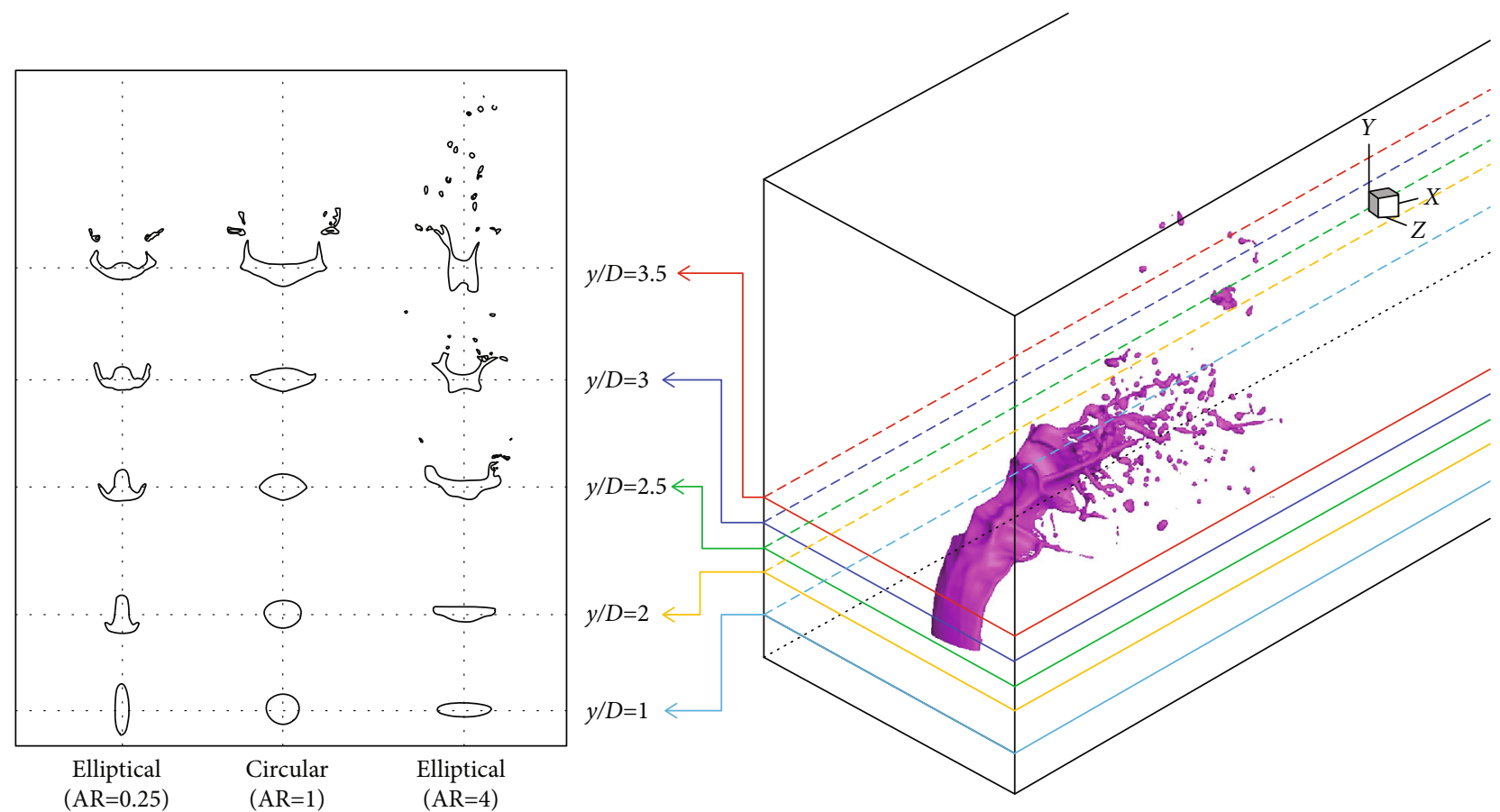

Figure 6: Cross-sectional shapes at $y / D=2,2.5$, and 3 for Case 1, Case 3, and Case 5, respectively, $t=56.4 \mu$ s.

3.2. Spatial Distribution of Elliptical Jets. To obtain the penetration depth of the elliptical liquid jet clearly and accurately, we extracted 22 transient spray images at $z=0$ section and superimposed them in Figure 8 . In the primary breakup stage, the larger $\mathrm{AR}$ is, the lower the penetration depth is. The reason is that the length vertical to the gas flow direction becomes longer with the increase of the aspect ratio. The windward area of the jet becomes larger, and the drag force of the crossflow to the jet becomes larger; thus, more droplets were stripped from both sides of the liquid column. The penetration depth of the jet becomes lower due to more liquid mass loss derived from more droplet stripping. The result is consistent with the conclusion in subsonic flow in [43]. The penetration depth of the elliptical liquid jet $(\mathrm{AR}=0.25)$ is $20 \%$ higher than the round liquid jet in Figure 9.

The spreading angle is the next significant spray characteristics. The spreading angle is defined in the upper right corner in Figure 10. Under the two-dimensional top view, the liquid drops from the center of the spray hole to the outermost edges of the left and right sides are straight lines, and the clamped angle is the spreading angle [43]. Figure 10 shows the spread angles at five different aspect ratios. The round jet has the widest spread angle which is $50.2^{\circ}$. Through the analysis of Figure 6, we can better understand the reason for the large spray spreading angle of the round jet. Apparently, the surface breakup of the round jet occurs later, so it maintains a bigger windward area. The further the droplets splash, the wider the spreading angle is. Compared with the round jet, the droplets of elliptical jets move away through the axial direction fragment from the sides.

Another index of atomization quality is the breakup length which is divided into the breakup length in the $x$ direction $\left(\mathrm{CBL}_{x}\right)$ and the breakup length in the $y$ direction $\left(\mathrm{CBL}_{y}\right)$.
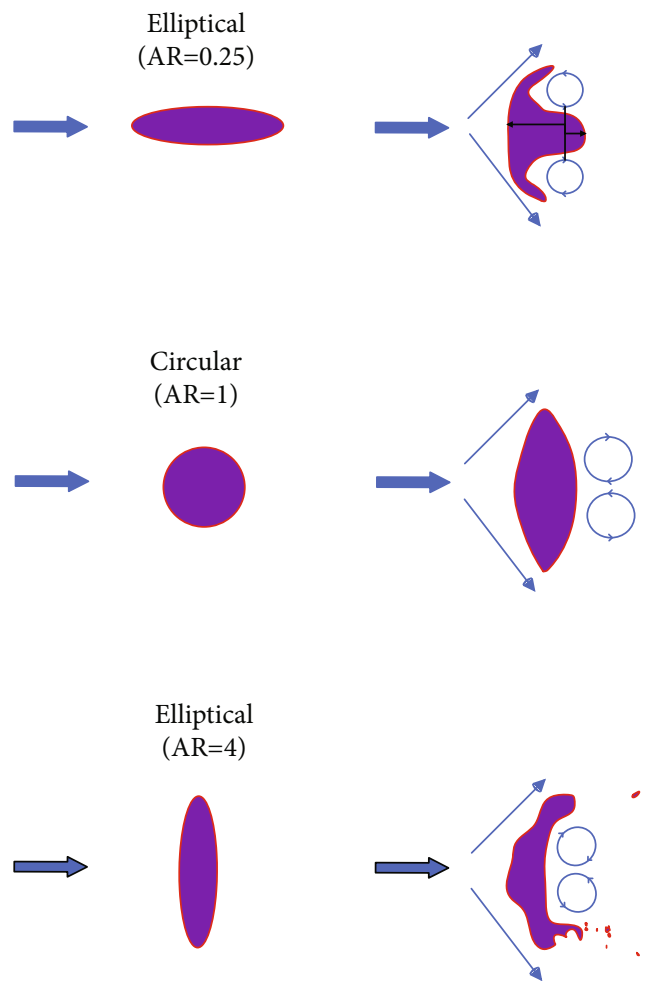

FIgURE 7: Breakup of elliptical liquid jets of three aspect ratios.

The breakup length represents the speed of the breakup of the liquid jet in the gas flow. Figure 11 shows that the isosurface $(\varphi=0)$ of the central section was extracted from the simulation results, and the liquid column breakup position was defined. The changing trend of the breakup 


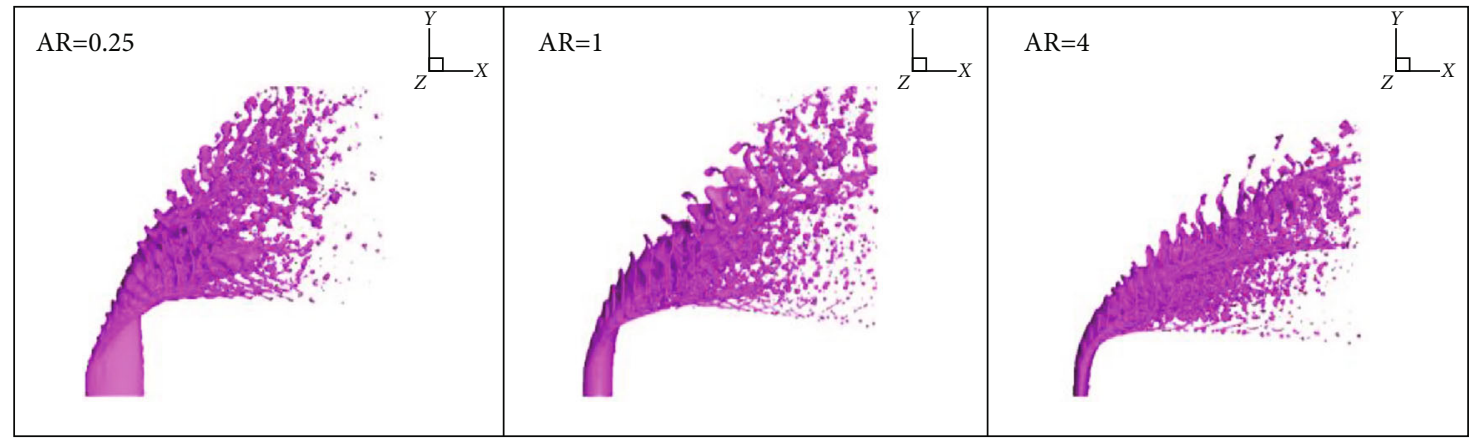

FIGURE 8: Spray structure of the liquid jet in different AR.

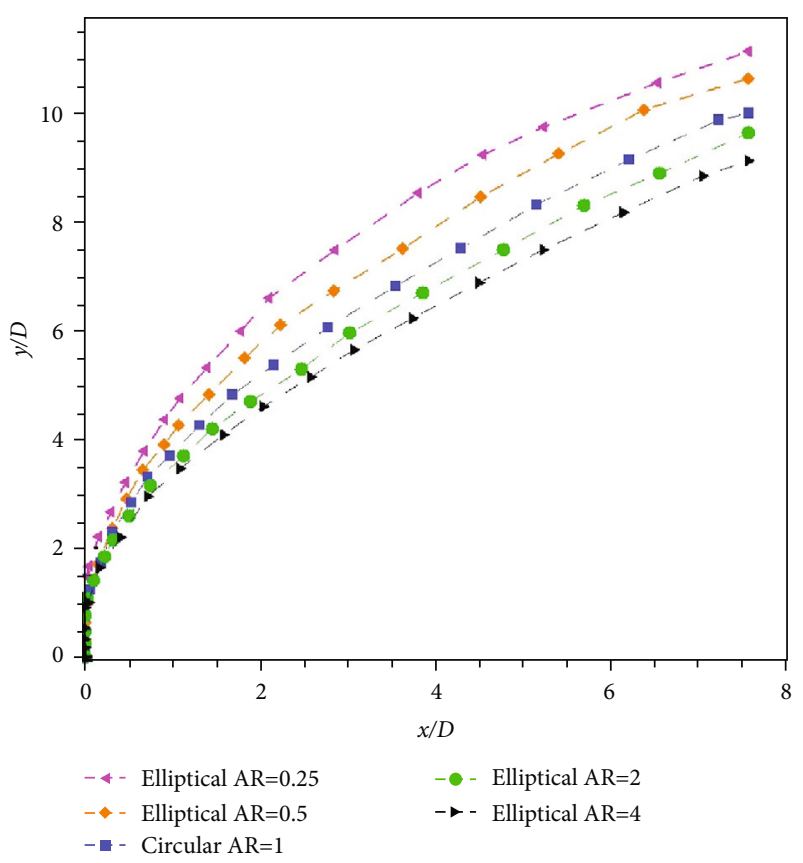

Figure 9: Penetration depth of the liquid jet at different AR.

length between the $x$ direction and $y$ direction is basically similar. Apparently, in the cases $(A R<1)$, the breakup length increases with the rise of $A R$, and the elliptical jet $(\mathrm{AR}=0.25)$ has the minimum breakup length in both directions, which is $59.2 \%$ lower than the round in the $x$ direction, $18.8 \%$ lower than the round in the $y$ direction, respectively. In the cases $(A R>1)$, the breakup length increases first and then decreases slightly.

3.3. Flow Field Characteristics of Elliptical Jet and Round Jet. Figure 12 presents a difference of the instantaneous vortex structures at three different AR. The column and surface breakup are observed at three AR at the same time. Compared with the round jet, there are more vortex structures in different scales near the location of surface breakup from the top left corner of Figure 12. The length of surface breakup in the $y$ direction is lower, which leads to the vortex structure here being lower. As the aspect ratio increases, the blocking effect of the liquid jet on the crossflow is enhanced gradually. The induced horseshoe vortex structure is larger and becomes further away from the liquid column.

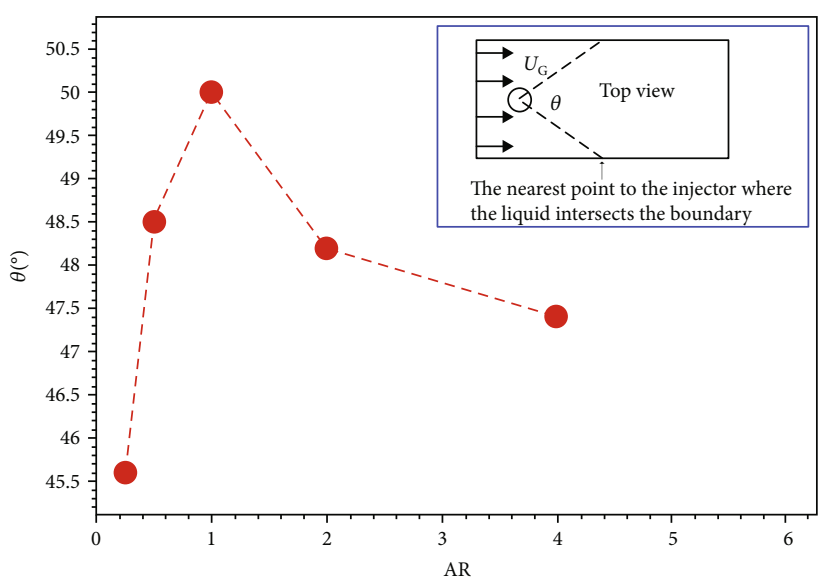

FIgURE 10: Spray spread angle of the round and elliptical orifice sprays.

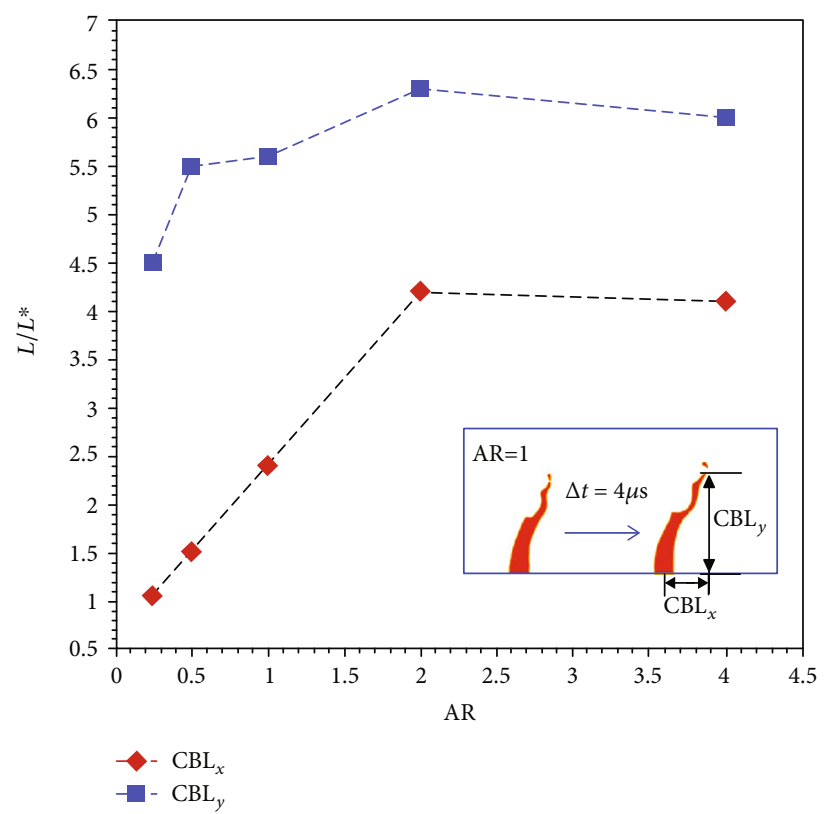

Figure 11: Liquid column breakup position and breakup length.

The dispersion of liquid droplets is determined by the large-scale vortices in the wake region which affects the mixing process of the spray deeply in the supersonic air flow in [35]. Figure 13 presents the vorticity distributions in slice 


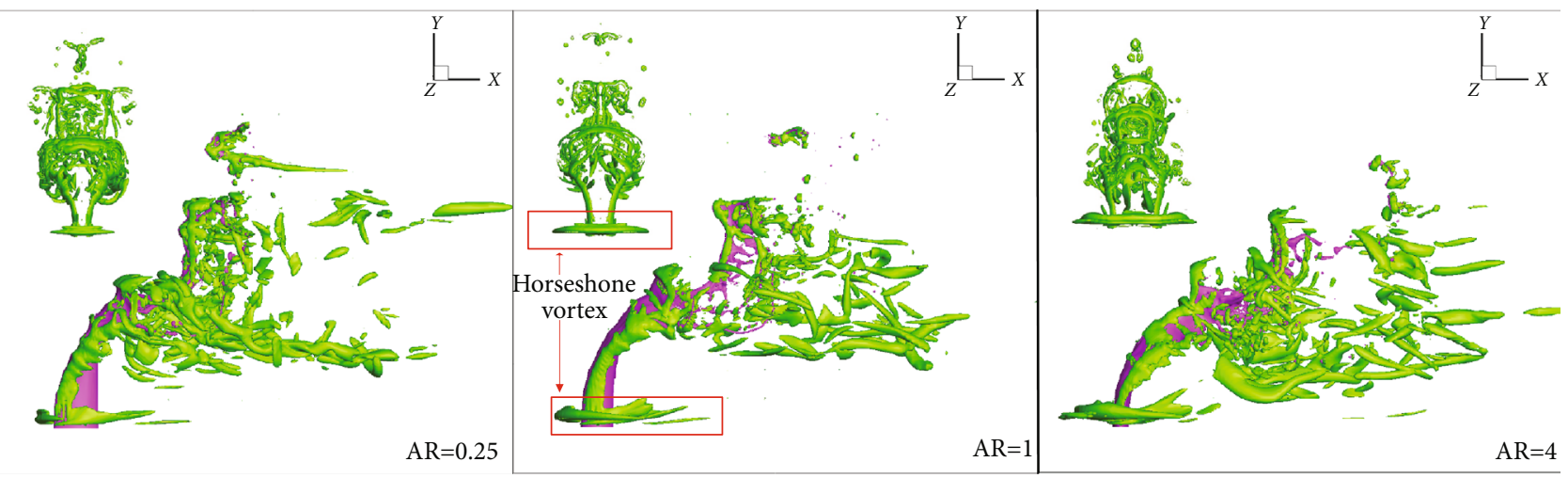

FIGURE 12: Instantaneous vortex structures in supersonic crossflow identified by isosurface of $Q, t=56.4 \mu \mathrm{s}$.

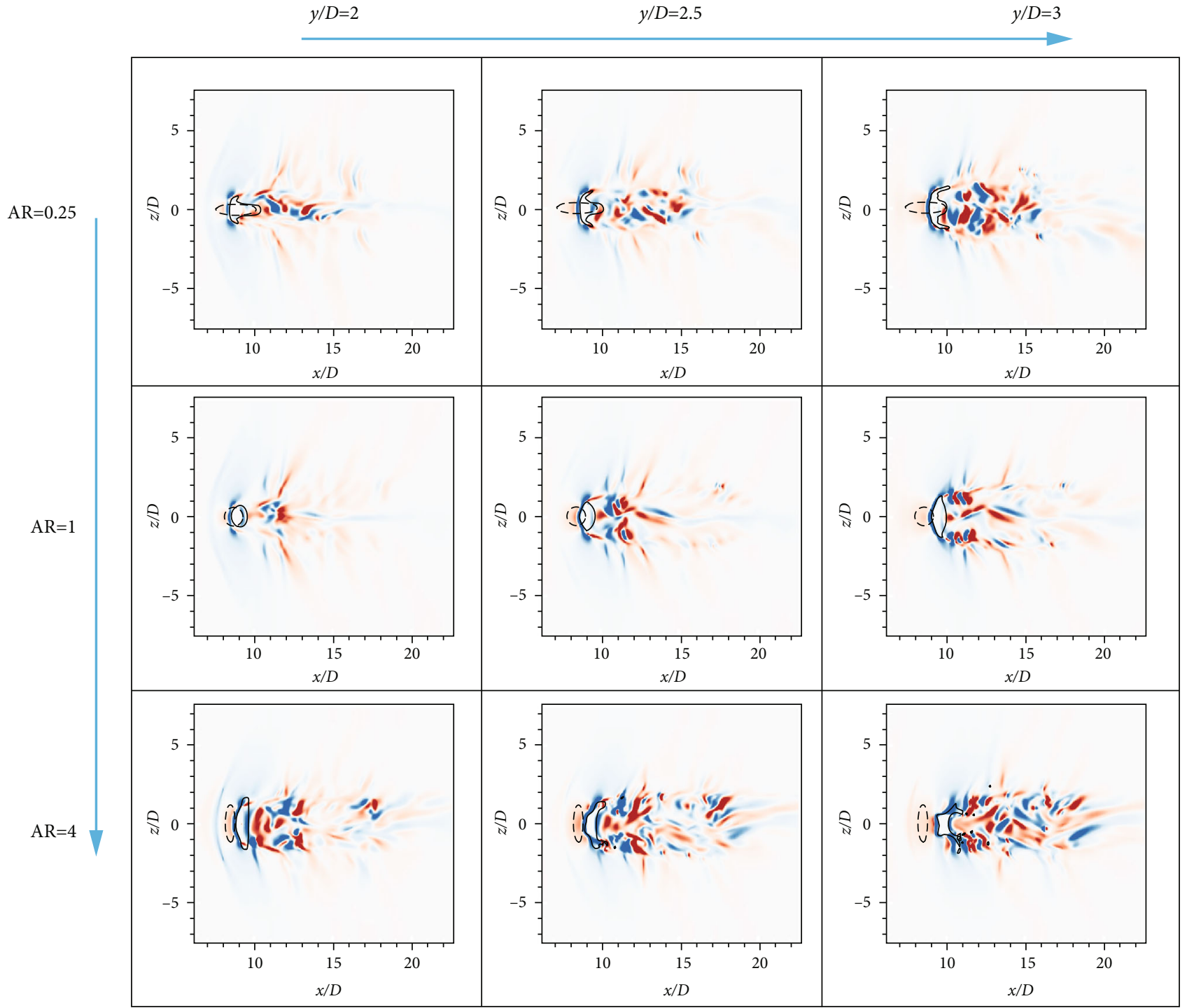

Figure 13: Vorticity distributions at $y / D=2,2.5$, and 3 for Case 1, Case 3, and Case 5.

$y / D=2,2.5$, and 3 for Case 1 , Case 3 , and Case 5 , respectively. Anticlockwise vortexes and clockwise vortexes are shown in blue and red, respectively. The wake region of the elliptical liquid jet $(\mathrm{AR}=4)$ is $18.4 \%$ larger than the round liquid jet.
The width of the wake region directly is reflected by the width of the windward side of the liquid column, which will change continuously due to the continuous breaking of the liquid column. The reason is that the occurrence of the surface 


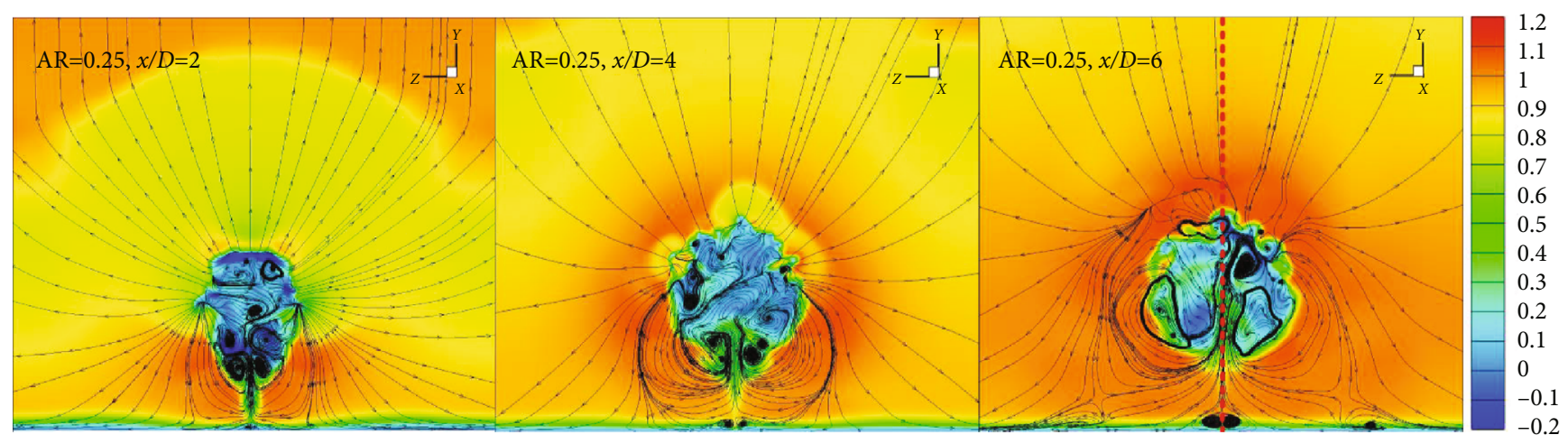

(a) Case 1

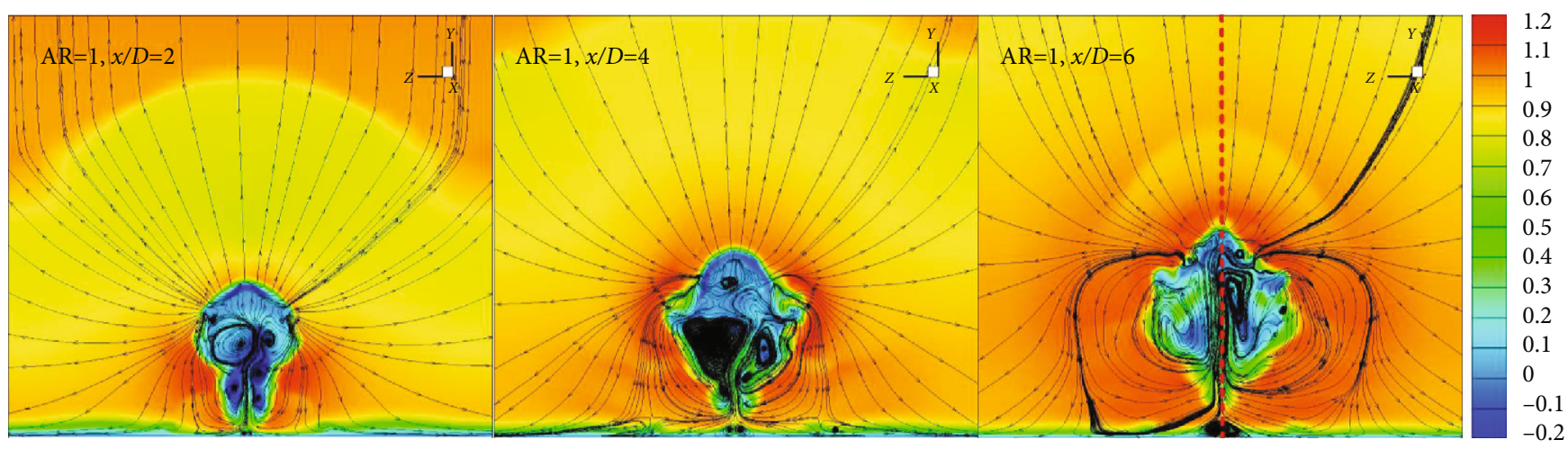

(b) Case 3

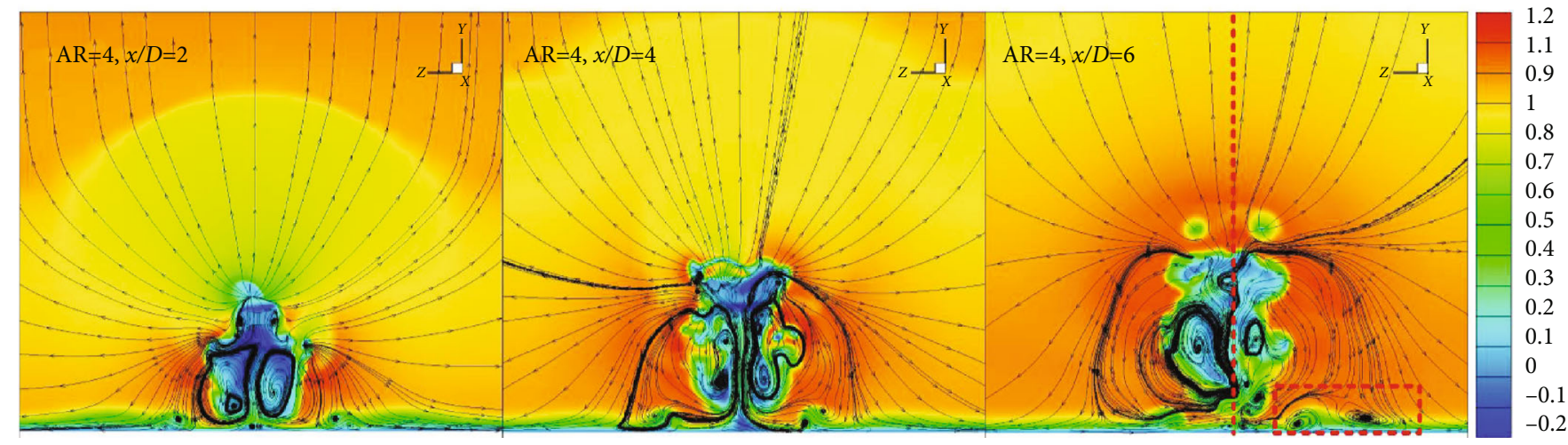

(c) Case 5

Figure 14: Streamlines with $U^{*}$ contours in slice $x / D=2$, 4 , and 6 for three AR, $t=56.4 \mu \mathrm{s}$.

break is difficult to be synchronized, so there is a time difference when the gas goes through the column.

Figure 14 shows the two-dimensional streamlines at different slices, i.e., $x / D=2,4$, and 6 with the contours of the nondimensionalized $x$ direction velocity $\left(U^{*}\right)$ for three AR of $0.25,1$, and 4 . Two pairs of reversal vortex pairs with obvious characteristics can be found under all working conditions. The first vortex pair located at the leeward side is relatively higher in height. The location of the vortex pairs becomes higher with the decrease of the aspect ratio due to the higher penetration depth. Furthermore, the vortex pairs will expand gradually with the increase of the flow distance. The direction of this vortex is straight up in the center line. The second vortex pairs located near the bottom wall have a nice symmetry. But, there is a certain offset from the center line in the case $(A R=4)$. The liquid column has the largest windward area in this condition and breaks up fastest. The time of the surface breakup on both sides of the column is out of sync.

A three-dimensional liquid jet structure in space is presented in Figure 15. After the supersonic flow is obstructed by the liquid column, a separation zone is forming located ahead of the column represented by the green circle. The gas flows into the separation zone and forms a pair of horseshoe vortex represented by the purple circle. The red transparent region represents the bow shock. Furthermore, the green squares indicate the column breakup position and surface breakup position, respectively. It can be seen that two 


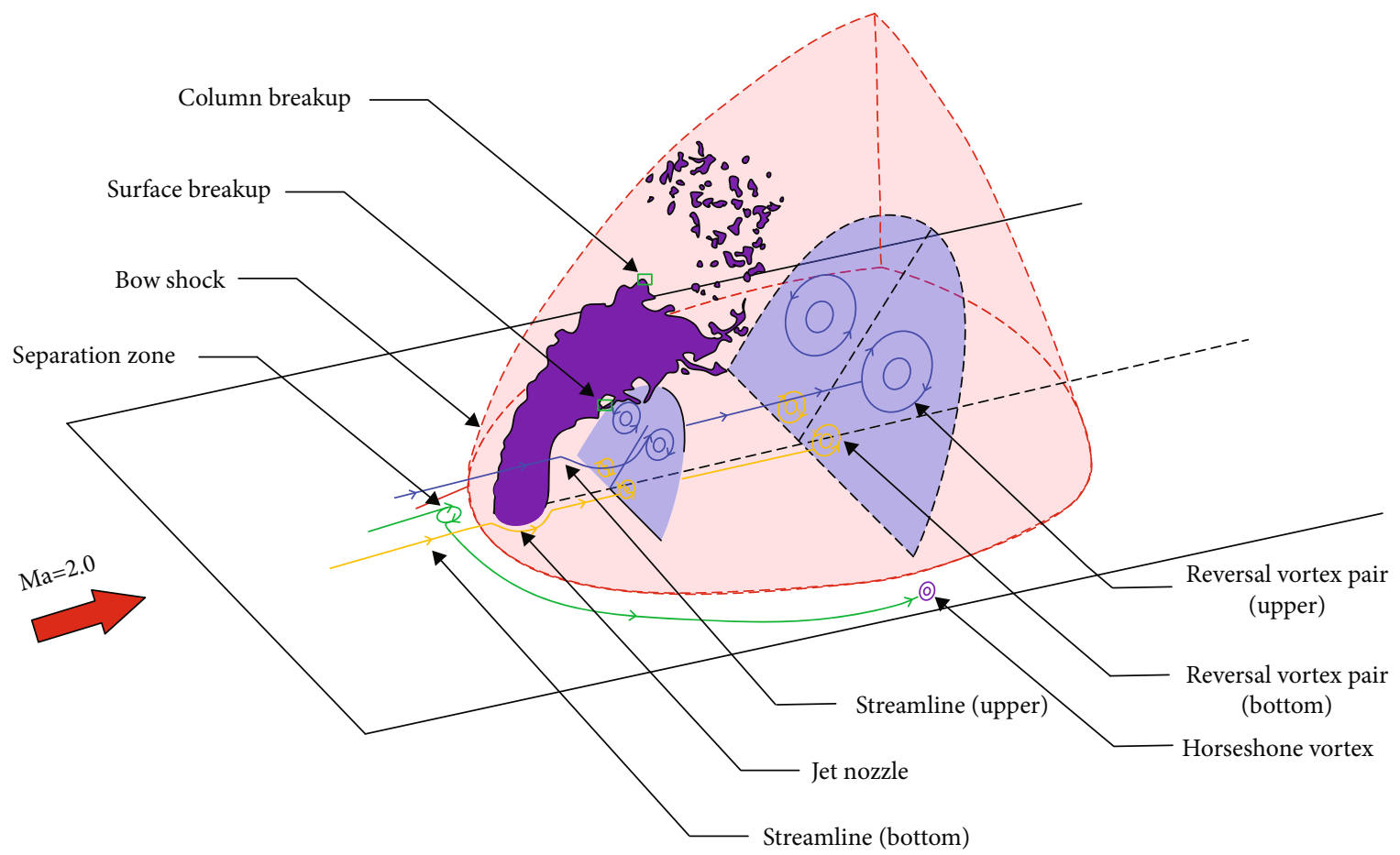

FIgURE 15: The flow field structure of the elliptical liquid jet in supersonic crossflow.

sections are taken in turn along the direction of the gas flow which is represented by dark blue, and two pairs of reverse vortex pairs are indicated by blue and orange circles. The vortex pairs become larger and larger gradually with the increases of the distance of gas flow.

\section{Conclusions}

In the current study, the primary breakup of a liquid jet of different orifices has been evaluated numerically. The aspect ratios are set to be $0.25,0.5,1,2$, and 4 . The spatial distribution including the changes of the jet's cross-section, liquid penetration depth, spreading angle, and breakup length has been compared at different aspect ratios. The flow field characteristics of the elliptical jet and round jet have been analyzed through the simulations. We have come to the following conclusions:

(1) Under the condition of supersonic crossflow, the surface breakup dominated by the R-T instability occurs earlier than that of the round liquid jet. The main reason is that the initial segment of the liquid column has different lengths in both the flow direction and spanwise direction. This result breaks the sequence and dominant degree of the $\mathrm{K}-\mathrm{H}$ instability with the R-T instability in the round orifice indirectly and promotes the occurrence of the surface breakup, so the surface breakup of the elliptical orifice jet occurs earlier than the round liquid jet. As a result, elliptical orifices can shorten the time of the surface breakup effectively
(2) In the liquid jet spatial distribution, the jet has a higher penetration depth at a larger aspect ratio normally. The penetration depth of the elliptical orifice with $\mathrm{AR}=0.25$ is $20 \%$ higher than the round orifice. When the jet orifice is round, the maximum spreading angle is 50.2. The windward area of the jet becomes larger, and the resistance of the crossflow to the jet becomes larger. The jet becomes more curved, so the penetration depth is smaller. At the same time, the surface breakup of the elliptical orifice jet occurs earlier than the round orifice; the round orifice can maintain a larger windward area in the downstream area of primary breakup, so it has the largest spreading angle. Therefore, the round orifice can enable the spray to reach a moderate penetration depth and a maximum spreading angle

(3) The changing trends of the breakup length of the jet in both directions are basically similar. The $\mathrm{CBL}_{x}$ $(\mathrm{AR}=0.25)$ is $59.2 \%$ lower than the round orifice, and the breakup length in the $y$ direction is $18.8 \%$ lower than the round orifice, resulting in the smaller the aspect ratio is, the smaller the windward area is. The greater the aerodynamic force on the unit area of the windward surface of the jet is, the more prone the jet column is to liquid column breakup, so the breakup length is smaller

(4) After analyzing the flow field characteristics of the liquid jet, it is found that the horseshoe vortex structure in front of the jet increases with the increase of the aspect ratio and becomes further away from the 
liquid column. The width of the wake area of the elliptical orifice $(A R=4)$ is $18.4 \%$ larger than that of the round orifice. Secondly, the surface breakup on both sides of the elliptical orifice liquid jet is generally difficult to synchronize. Two pairs of reversal vortex pairs with obvious characteristics can be found under all working conditions. The first vortex pair located at the leeward side is relatively higher in height. The location of the vortex pair becomes higher with the decrease of the aspect ratio due to the higher penetration depth. Furthermore, the vortex pairs will expand gradually with the increase of the flow distance. The direction of this vortex is straight up in the center line. The second vortex located near the bottom wall has a nice symmetry. The time of the surface breakup on both sides of the column is out of sync. After the airflow passes through the surface breakup position, there is a time difference when it flows into the rear of the liquid column, which eventually leads to the asymmetric center offset vortex pairs behind the liquid column different from the round liquid jet

\section{Nomenclature}

$\begin{array}{ll}\text { AR: } & \text { Orifice cross-section aspect ratio }(b / a) \\ \text { CBL }_{x}: & \text { Breakup length in the } x \text { direction } \\ \text { CBL }_{y}: & \text { Breakup length in the } y \text { direction } \\ \text { CLSVOF: } & \text { Coupled level set and volume of fluid } \\ D: & \text { Equivalent liquid jet diameter } \\ F: & \text { Liquid volume fraction } \\ \text { LS: } & \text { Level set } \\ \text { Ma: } & \text { Mach number } \\ P_{\mathrm{G}}: & \text { Gas static pressure } \\ P_{\mathrm{L}}: & \text { Water pressure } \\ P^{*}: & \text { Nondimensionalized pressure } P /\left(\rho_{G} U_{G}^{2}\right) \\ Q: & \text { Second invariance of velocity gradient tensor } \\ T_{\mathrm{G}}: & \text { Temperature } \\ U_{\mathrm{L}}: & \text { Liquid density } \\ U_{\mathrm{G}}: & \text { Gas velocity } \\ U^{*}: & \text { Nondimensionalized } x \text { direction velocity } U / U_{G} \\ \mathrm{VOF}: & \text { Volume of fluid } \\ a: & \text { Orifice diameter parallel to the gas flow } \\ b: & \text { Orifice diameter normal to the gas flow } \\ q: & \text { Liquid/gas momentum flux ratio } \\ \lambda: & \text { Surface wavelength } \\ \mu_{\mathrm{G}}: & \text { Gas viscosity } \\ \mu_{\mathrm{L}}: & \text { Liquid viscosity } \\ \rho_{\mathrm{G}}: & \text { Gas density } \\ \rho_{\mathrm{L}}: & \text { Liquid density } \\ \sigma: & \text { Surface tension } \\ \varphi: & \text { Level set function } \\ \text { We } & \text { Relative gas-liquid Weber number } \rho_{\mathrm{G}} U_{\mathrm{G}}^{2} D_{\mathrm{eq}} / \sigma . \\ & \end{array}$

\section{Data Availability}

The datasets generated during and/or analyzed during the current study are available from the corresponding author on reasonable request.

\section{Conflicts of Interest}

The authors declare that they have no conflicts of interest.

\section{Acknowledgments}

This work was supported by National Natural Science Foundation of China (grant numbers 11872375 and 11902351).

\section{References}

[1] E. A. Kush and J. A. Schetz, "Liquid jet injection into a supersonic flow," AIAA Journal, vol. 11, no. 9, pp. 1223-1224, 1973.

[2] J. A. Schetz, E. A. Kush, and P. B. Joshi, "Wave phenomena in liquid jet breakup in a supersonic crossflow," AIAA Journal, vol. 18, no. 7, pp. 774-778, 1980.

[3] K.-C. Lin, P. Kennedy, and T. Jackson, "Structures of water jets in a Mach 1.94 supersonic crossflow," in Proceedings of the 42nd AIAA Aerospace Sciences Meeting and Exhibit, Reno, Nevada, January 2004.

[4] C. Li, C. Li, F. Xiao, Q. Li, and Y. Zhu, "Experimental study of spray characteristics of liquid jets in supersonic crossflow," Aerospace Science and Technology, vol. 95, p. 105426, 2019.

[5] L. Y. Wu, Z. G. Wang, Q. Li, and C. Li, "Study on transient structure characteristics of round liquid jet in supersonic crossflows," Journal of Visualization, vol. 19, no. 3, pp. 337341, 2016.

[6] Z. G. Wang, L. Wu, Q. Li, and C. Li, "Experimental investigation on structures and velocity of liquid jets in a supersonic crossflow," Applied Physics Letters, vol. 105, no. 13, article 134102, 2014.

[7] J. B. Perurena, C. O. Asma, R. Theunissen, and O. Chazot, "Experimental investigation of liquid jet injection into Mach 6 hypersonic crossflow," Experiments in Fluids, vol. 46, no. 3, pp. 403-417, 2009.

[8] P. K. Wu, K. A. Kirkendall, R. P. Fuller, and A. S. Nejad, "Breakup processes of liquid jets in subsonic crossflows," Journal of Propulsion and Power, vol. 13, no. 1, pp. 64-73, 1997.

[9] F. Jabbari, M. Jadidi, R. Wuthrich, and A. Dolatabadi, “A numerical study of suspension injection in plasma-spraying process," Journal of Thermal Spray Technology, vol. 23, no. 12, pp. 3-13, 2014.

[10] M. Jadidi, S. Moghtadernejad, and A. Dolatabadi, "A comprehensive review on fluid dynamics and transport of suspension liquid droplets and particles in high-velocity oxygen-fuel (HVOF) thermal spray," Coatings, vol. 5, no. 4, pp. 576-645, 2015.

[11] S.-Y. No, "A review on empirical correlations for jet/spray trajectory of liquid jet in uniform cross flow," International Journal of Spray and Combustion Dynamics, vol. 7, no. 4, pp. 283-313, 2015.

[12] M. Jadidi, S. Moghtadernejad, and A. Dolatabadi, "Penetration and breakup of liquid jet in transverse free air jet with application in suspension-solution thermal sprays," Materials \& Design, vol. 110, pp. 425-435, 2016.

[13] M. Jadidi, S. Moghtadernejad, and A. Dolatabadi, "Numerical simulation of primary breakup of round nonturbulent liquid jets in shear-laden gaseous crossflow," Atomization and Sprays, vol. 27, no. 3, pp. 227-250, 2017. 
[14] K. Lee, C. Aalburg, F. J. Diez, G. M. Faeth, and K. A. Sallam, "Primary breakup of turbulent round liquid jets in uniform crossflows," AIAA Journal, vol. 45, no. 8, pp. 1907-1916, 2007.

[15] K. A. Sallam, C. Aalburg, and G. M. Faeth, "Breakup of round nonturbulent liquid jets in gaseous crossflow," AIAA Journal, vol. 42, no. 12, pp. 2529-2540, 2004.

[16] J. Song, K. Ahn, M.-K. Kim, and Y. Yoon, "Effects of orifice internal flow on liquid jets in subsonic crossflows," Journal of Propulsion and Power, vol. 27, no. 3, pp. 608-619, 2011.

[17] M. Herrmann, "Detailed numerical simulations of the primary atomization of a turbulent liquid jet in crossflow," Journal of Engineering for Gas Turbines and Power, vol. 132, no. 6, 201061506-61510.

[18] S. E. Bechtel, J. A. Cooper, M. G. Forest et al., "A new model to determine dynamic surface tension and elongational viscosity using oscillating jet measurements," Journal of Fluid Mechanics, vol. 293, pp. 379-403, 1995.

[19] T. V. Kasyap, D. Sivakumar, and B. N. Raghunandan, "Flow and breakup characteristics of elliptical liquid jets," International Journal of Multiphase Flow, vol. 35, no. 1, pp. 8-19, 2009.

[20] G. Amini and A. Dolatabadi, "Axis-switching and breakup of low-speed elliptic liquid jets," International Journal of Multiphase Flow, vol. 42, pp. 96-103, 2012.

[21] P. Sharma and T. Fang, "Breakup of liquid jets from noncircular orifices," Experiments in Fluids, vol. 55, no. 2, p. 1666, 2014.

[22] F. Wang and T. Fang, "Liquid jet breakup for non-circular orifices under low pressures," International Journal of Multiphase Flow, vol. 72, pp. 248-262, 2015.

[23] M. Marzbali, Penetration of circular and elliptical liquid jets into gaseous crossflow, Doctoral dissertation, Concordia University, 2011.

[24] M. R. Morad and H. Khosrobeygi, "Penetration of elliptical liquid jets in low-speed crossflow," Journal of Fluids Engineering, vol. 141, no. 1, 2019011-301.

[25] D. H. Fontes, V. Vilela, L. Souza Meira, and F. José de Souza, "Improved hybrid model applied to liquid jet in crossflow," International Journal of Multiphase Flow, vol. 114, pp. 98114, 2019.

[26] N. Liu, Z. G. Wang, M. B. Sun, R. Deiterding, and H. Wang, "Simulation of liquid jet primary breakup in a supersonic crossflow under adaptive mesh Refinement framework," Aerospace Science and Technology, vol. 91, pp. 456-473, 2019.

[27] C. H. Chang, X. Deng, and T. G. Theofanous, "Direct numerical simulation of interfacial instabilities: a consistent, conservative, all-speed, sharp-interface method," Journal of Computational Physics, vol. 242, pp. 946-990, 2013.

[28] J. C. Meng and T. Colonius, "Numerical simulation of the aerobreakup of a water droplet," Journal of Fluid Mechanics, vol. 835, pp. 1108-1135, 2018.

[29] F. Xiao, Z. G. Wang, M. B. Sun, N. Liu, and X. Yang, "Simulation of drop deformation and breakup in supersonic flow," Proceedings of the Combustion Institute, vol. 36, no. 2, pp. 2417-2424, 2017.

[30] D. P. Garrick, W. A. Hagen, and J. D. Regele, "An interface capturing scheme for modeling atomization in compressible flows," Journal of Computational Physics, vol. 344, pp. 260280, 2017.

[31] D. P. Garrick, M. Owkes, and J. D. Regele, "A finite-volume hllc-based scheme for compressible interfacial flows with sur- face tension," Journal of Computational Physics, vol. 339, pp. 46-67, 2017.

[32] V. B. Betelin, N. N. Smirnov, V. F. Nikitin, V. R. Dushin, A. G. Kushnirenko, and V. A. Nerchenko, "Evaporation and ignition of droplets in combustion chambers modeling and simulation," Acta Astronautica, vol. 70, pp. 23-35, 2012.

[33] P. B. Li, Z. G. Wang, X. S. Bai et al., "Three-dimensional flow structures and droplet-gas mixing process of a liquid jet in supersonic crossflow," Aerospace Science and Technology, vol. 90, pp. 140-156, 2019.

[34] P. B. Li, Z. G. Wang, M. B. Sun, and H. Wang, "Numerical simulation of the gas-liquid interaction of a liquid jet in supersonic crossflow," Acta Astronautica, vol. 134, pp. 333-344, 2017.

[35] Y. H. Zhu, F. Xiao, Q. L. Li, R. Mo, C. Li, and S. Lin, "Les of primary breakup of pulsed liquid jet in supersonic crossflow," Acta Astronautica, vol. 154, pp. 119-132, 2019.

[36] M. Sussman and E. G. Puckett, "A coupled level set and volume-of-fluid method for computing $3 \mathrm{D}$ and axisymmetric incompressible two-phase flows," Journal of Computational Physics, vol. 162, no. 2, pp. 301-337, 2000.

[37] F. Xiao, Large eddy simulation of liquid jet primary breakup, $\mathrm{PhD}$, Loughborough University, 2012.

[38] F. Xiao, Z. G. Wang, M. B. Sun, J. H. Liang, and N. Liu, "Large eddy simulation of liquid jet primary breakup in supersonic air crossflow," International Journal of Multiphase Flow, vol. 87, no. 12, pp. 229-240, 2016.

[39] F. Xiao, M. Dianat, and J. J. McGuirk, "A robust interface method for drop formation and breakup simulation at high density ratio using an extrapolated liquid velocity," Computers \& Fluids, vol. 136, pp. 402-420, 2016.

[40] R. P. Fedkiw, T. Aslam, B. Merriman, and S. Osher, “A nonoscillatory eulerian approach to interfaces in multimaterial flows (the ghost fluid method)," Journal of Computational Physics, vol. 152, no. 2, pp. 457-492, 1999.

[41] X. D. Liu, R. P. Fedkiw, and M. Kang, "A boundary condition capturing method for poisson's equation on irregular domains," Journal of Computational Physics, vol. 160, no. 1, pp. 151-178, 2000.

[42] G. S. Jiang and C. W. Shu, "Efficient implementation of weighted eno schemes," Journal of Computational Physics, vol. 126, no. 1, pp. 202-228, 1996.

[43] E. Farvardin and A. Dolatabadi, "Breakup simulation of elliptical liquid jet in gaseous crossflow," in 42nd AIAA Fluid Dynamics Conference and Exhibit, New Orleans, Louisiana, June 2012. 